\title{
Coulisses
}

Revue de théâtre

25 | Hiver 2002

Varia

\section{Roman de familles, une méthode d'écriture}

Entretien avec Christophe Merlant, assistant à la conception

Lucile Garbagnati

\section{CpenEdition}

Journals

Édition électronique

URL : http://journals.openedition.org/coulisses/5928

DOI : $10.4000 /$ coulisses.5928

ISSN : 2546-9460

Éditeur

Presses universitaires de Franche-Comté

\section{Édition imprimée}

Date de publication : 1 janvier 2002

Pagination : 24-29

ISBN : 2-84627-052-X

ISSN : $1150-594 \mathrm{X}$

Référence électronique

Lucile Garbagnati, «Roman de familles, une méthode d'écriture », Coulisses [En ligne], 25 | Hiver 2002, mis en ligne le 24 octobre 2019, consulté le 15 novembre 2019. URL : http://journals.openedition.org/ coulisses/5928; DOI : 10.4000/coulisses.5928

Ce document a été généré automatiquement le 15 novembre 2019.

Coulisses 


\title{
Roman de familles, une méthode d'écriture
}

\author{
Entretien avec Christophe Merlant, assistant à la conception
}

Lucile Garbagnati

Conception et mise en scène Alain Mollot, assistant à la conception Christophe Merlant Coproduction: La Coupole, Scène nationale de Sénart, La Comédie de Picardie, Le Channel, Scène nationale de Calais, L'Allan, Scène nationale de Montbéliard, Nouveau Théâtre de Besançon, Centre dramatique national, Théâtre Romain Rolland, Villejuif Représentations à Besançon, 6, 7, et 8 novembre

L. G. - dans Ce spectacle vous Êtes " ASsistant À la Conception ", Cela consiste EN QUOI?

C. M. - Effectivement on a inventé cette expression parce que le type de travail que j'ai fait dans l'itinéraire des spectacles aboutissant à Roman (hormis deux où je ne suis pas intervenu) est assez particulier. Il ne s'agit ni de dramaturgie au sens brechtien, puisque nous n'avons pas voulu introduire un sens autre, une « idéologie » ou un jugement sur la parole des gens, ni un travail d'écriture proprement dite puisqu'il s'agit de porter la parole des gens vers le théâtre. C'est donc un travail d'assistant à la conception, un peu comme un travail de sage-femme, une conception c'est bien une naissance, mais ce n'est pas la sage-femme qui crée.

\section{G. - PRATIQUement COMMEnt AVEZ-VOUS PROCÉdÉ ?}

C. M. - Il y a plusieurs strates d'interventions. Tout d'abord tout un renvoi de lectures sur le lien familial et de conversations sur les lectures avec Mollot. Découvrir des textes, comme par exemple les Nourritures affectives de Cirulnik, ou les textes de l'École de Palo Alto sur les problèmes de la communication dans le lien familial. Depuis longtemps on se renvoyait des choses dans ce domaine, et surtout depuis un travail d'adaptation que nous avions fait avec Wieslaw Komassa sur La Mère de Wietkiewitz. On ne sait jamais d'où vient l'impérieuse nécessité, ni exactement par quelles voies un metteur en scène l'éprouve, mais on peut dire que Mollot le portait depuis longtemps et que ça s'est imposé à un moment comme une évidence, de s'avancer et de perdurer dans cette aventure de deux ans. 


\section{G. - COMMENT VOUS ÊTES-VOUS MIS À LA TÂCHE ?}

C. M. - On a un peu inventé la méthode en cours de création. Il y a d'abord eu le défi lancé par Jacques Livchine lors de Ah Montbélamour ${ }^{1}$ ! Elsa Quinette recueillait les interviews. Elle vient de la radio, et moi j'avais plus d'expérience dans le domaine de la presse écrite - le texte et la photo - et une longue habitude du travail avec Mollot. À la longue, on finit par savoir ce qu'il faut comme matériau de départ pour inspirer un metteur en scène qui est de la famille de ceux qui pratiquent un théâtre du personnage du geste et de l'image... Mais ces catégories sont réductrices et je les utilise avec des pincettes... Donc de longues conversations avec Elsa, de relance et d'ajustements pendant qu'elle faisait sa cueillette. Relancer sur telle situation, réorienter, tenter d'affiner sur telle anecdote qui semble significative, demander quels gestes, quels objets, etc. C'était passionnant et on peut dire qu'une certaine méthode pas un procédé s'est inventée pour organiser les rencontres avec les personnes qui parlaient. Cela nous a beaucoup servi par la suite à elle et à moi pour la suite des cueillettes...

\section{G. - VOUS DITES DES RENCONTRES POURQUOI PAS DES INTERVIEWS ?}

C. M. - Justement, il faut établir clairement des nuances essentielles dans ce type de travail. C'est grâce à ces nuances faites dès le départ qu'on peut recueillir la matière juste qui permet de travailler dans un certain style et une certaine dimension. Il ne s'agit pas d'une posture d'intervieweur ou d'enquêteur. L'intervieweur cherche le fait, la personne qui sort de l'ordinaire, ne serait-ce qu'un jour! Il y a toujours làdessous un peu de sensationnalisme médiatique. L'enquête c'est différent, c'est un rapport à la vérité, on recoupe, on établit les faits, il y a une dimension un peu policière ou historienne. Une rencontre, d'abord c'est un temps plus long, et une préoccupation différente. Ça se passe de personne à personne, dans la confiance. Donc ni interview, ni enquête ni récit de vie non plus. Le récit de vie a une visée thérapeutique ou d'adaptation... de restructuration de la personnalité. Pour faire bref il faut arriver à n'être ni journaliste, ni policier, ni psychiatre... ce sont des postures qu'on peut prendre insensiblement, mais qui ne donnent pas grand-chose pour ce qu'on cherchait... et qui orienteraient vers un autre type de théâtre.

\section{G. - Cela PARAît UN PEU MystérieuX et TENIR AU tALENT de CHACUN.}

C. M. - Bien sûr... mais le talent ça se prépare, ça se révèle et ça s'entretient. Concrètement dans ce travail de préparation à la rencontre il faut déjà "être " intéressé. Ça peut paraître trivial mais ça ne l'est pas. «Inter-esse » c'est être avec quelqu'un et mettre entre soi et l'autre quelque chose qui nous préoccupe, et qui ne soit pas simplement moi ou toi, ce qui dans le fond est de peu d'importance et d'intérêt, ou carrément ennuyeux et bavard. Ce qu'il y a d'intéressant c'est quand quelqu'un vous parle de l'Amour, de la Rencontre, de la Rupture ou du Deuil à travers son amour, son deuil... Quand une personne qui vous reçoit en disant «je suis ordinaire, je n'ai rien de bien exceptionnel à vous dire » arrive à quitter le fait divers, l'anecdote, elle peut prendre une dimension proprement tragique. Ça arrive de voir les gens se mettre carrément dans la verticalité du grand malheur ou du grand bonheur et quitter l'horizontalité de la conversation du petit échange... C'est un peu dur à expliquer mais ça se sent... Quand je forme des étudiants du DEUST de l'École de la marionnette, ou de la faculté des lettres à ce type de rencontre pour le théâtre, je choisis des lectures. Par exemple, quelques textes de Barthes, tirés des Fragments d'un discours amoureux, pour le travail qu'on avait mené sur l'événement frappant de la 
famille, À part ça tout va bien. Une fois en parlant de ces textes à une étudiante qui menait une rencontre, je lui demande si ça lui sert... Elle me répond : "Oui, mais ça m'intéresse sur le plan personnel surtout...» Elle avait oublié que c'est ce plan personnel qu'elle mettait en jeu dans la rencontre, celui d'une personne qui s'interroge avec Barthes, sur l'attente, les variations de l'amour, du bonheur et du malheur. Elle s'était intéressée, devenait intéressante et du coup cela rend intéressant celui qui est en face. Je me rappelle une boutade d'un de nos enseignants de l'école de journalisme de la rue du Louvre «La bonne interview c'est quand on commence à parler de soi...!" Il y a donc une préparation, ce que j'appelle « charger» la personne, comme on charge une pile ou une batterie. Ce n'est pas un procédé technique.

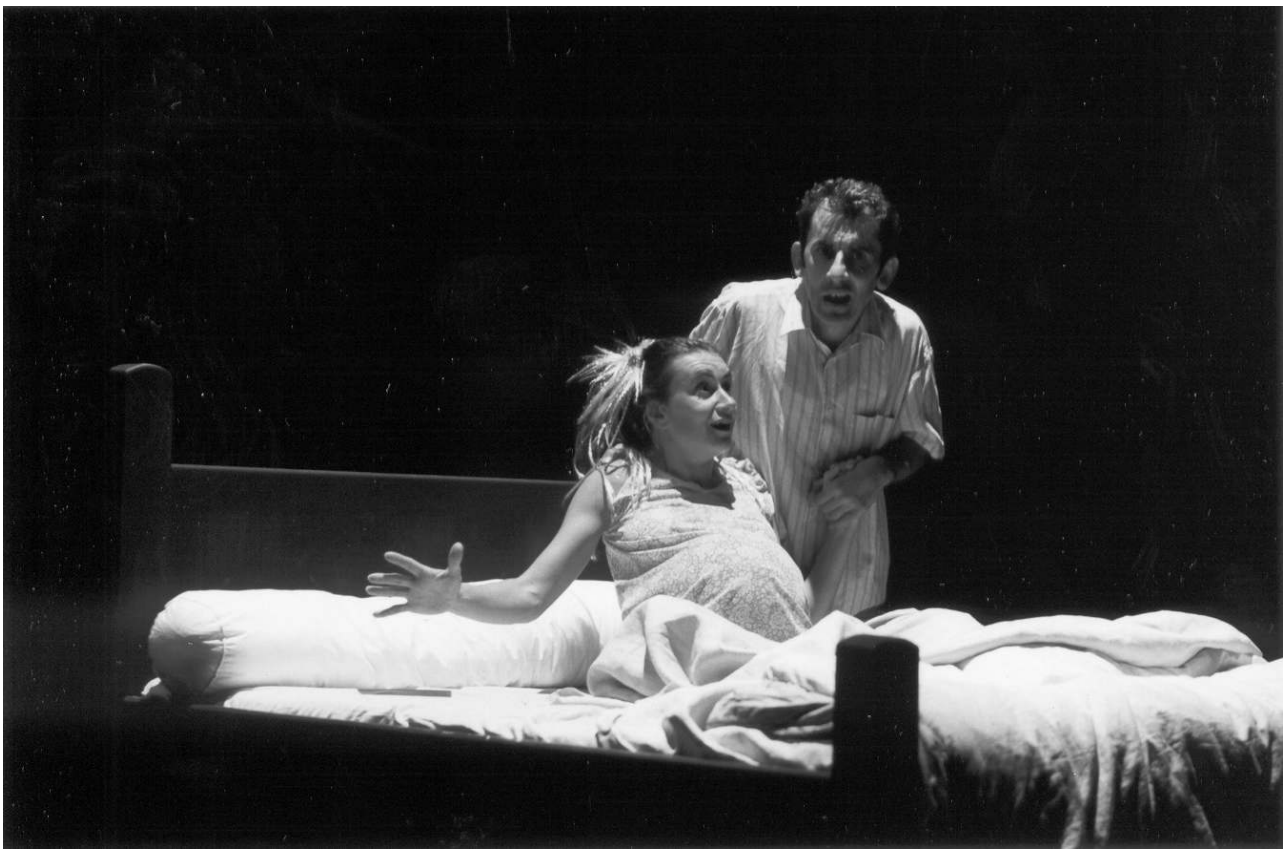

Théâtre de la Jacquerie

\section{G. - ET UNE FOIS LE MATÉRIAU RECUEILLI, COMMENT PROCÉDEZ-VOUS ?}

C. M. - Dans un premier temps Mollot sculpte avec des ciseaux pour papier... Il choisit en fonction de la situation, du personnage, il trie. C'est toujours difficile de trier. Mais là, c'est pas de la sculpture, c'est possible de revenir en arrière. Parfois j'interviens, on en discute, parfois non, parfois les étudiants quand c'était eux qui recueillaient ont proposé des premières coupes. Ça a été différent pour chaque spectacle, selon les urgences et les disponibilités.

\section{G. - MAIS QUAND LE TRAVAIL D'ÉCRITURE INTERVIENT-IL ?}

C. M. - L'expression est juste, c'est un travail d'écriture et non d'écrivain. Sans quoi ce ne serait plus la parole des gens, ce que nous recherchons. Je suis intervenu avec une gomme, d'ailleurs pas dans tous les spectacles de la saga ! Écrire avec une gomme c'est « faire du quatre phrase en une » dans notre jargon, ou mettre ici ce qui n'y était pas, mais qui a été dit. Tout est dit mais parfois dans le désordre ou dans un temps qui n'est pas le temps théâtral alors il faut réajuster. Chercher dans le discours de la personne les personnages qui s'y trouvent et les faire exister théâtralement. Mollot ne travaille pas dans une esthétique du document brut, on pense que le respect n'est pas du voyeurisme. C'est l'exercice périlleux qui consiste à la fois à ne pas se servir de 
l'autre pour le théâtre - ce serait une instrumentalisation odieuse, comme la tentation de la dérision - et à servir le théâtre. On reste dans l'optique du spectacle de théâtre, ce n'est pas de la mise en espace et en voix de témoignages. Dès le départ c'est clair avec les gens qui sont rencontrés, le contrat est clair, il faut être précis... et quand ils sont dans la salle ils ne se sentent pas trahis...

\section{G. - ON POURRAIT DONC DIRE QU'IL Y A DES STRATIFICATIONS D'ÉCRITURES...}

C. M. - Absolument, ça commence avant la rencontre, dans la préparation. Ensuite celui qui parle c'est bien un travail de mémoire, d'écriture de soi-même si c'est à l'oral. Et puis il y a l'écriture des grands choix, on s'achemine vers le théâtre, puis l'affinage des petits choix. Allons, alors, plus loin... L'écriture de la mise en scène, de la musique, de l'éclairage, et bien entendu la dernière visible celle du comédien qui interprète. C'est curieux parce que ce type de travail enclenche parfois le débat : estce qu'il peut y avoir du théâtre sans auteur ? Et parfois c'est pas un texte, il n'y a pas d'auteur, ou à l'inverse ça c'est ce qu'on cherche au théâtre, c'est de la parole vivante. Ce genre d'oppositions me semble assez stérile. Ça vient je pense d'une mythologie datant de l'époque romantique, celle de l'artiste comme "originalité individuelle absolue » qui perdure, l'avènement de la signature dans les arts, qui fait que l'on est plus sensible à la signature qu'à l'œuvre. Avec la surmédiatisation de la création et la transformation de l'artistique en produits culturels labellisés à la signature, on finit par vendre des signatures et à ne plus s'intéresser aux œuvres. Bref, le système tourne un peu en rond. Sans compter que par là-dessus, le commentaire sur l'œuvre devient aussi important que l'œuvre et sa réception par le public, par voie de " colloquisations ", manifestes journalistiques, petits scandales qui ne blessent que des amours-propres, et autres "produits dérivés " comme on dit au cinéma, qui vendent, théorisent, inventent des concepts, dressent des frontières, emboitent le pas à des révoltes contre des esthétiques officielles qui n'existent plus, etc. Moi je pense qu'il y a plusieurs manières de procéder dans la création. Ici il y a une écriture disons « polyphonique » mais « la mise en œuvre » de tout ça, c'est bien un acte de création. Bien sûr il y a eu une époque du «texte-roi » puis la période du "metteur en scèneroi » mais il me semble que désormais, et de plus en plus, parler du théâtre au singulier devient difficile... et sans grand intérêt. À la question: Une création collective mérite-t-elle le nom de création ? je réponds : on travaille en collectif, mais à un moment il y a une « mise en œuvre " qui tient à une personne. Il faut quelqu'un qui convoque les autres et oriente le tout. Que ce soit un auteur qui élise son metteur en scène et des comédiens, ou à l'inverse un metteur en scène qui s'adjoint un auteur ou des auteurs, ou des gens qui ne sont pas auteurs mais savent parler - tous ces cas de figure existent - peu importe à mon sens. Il y a des échecs et des réussites dans toutes ses configurations. Et heureusement, sans quoi ce serait plus de l'usinage que de l'artistique. 


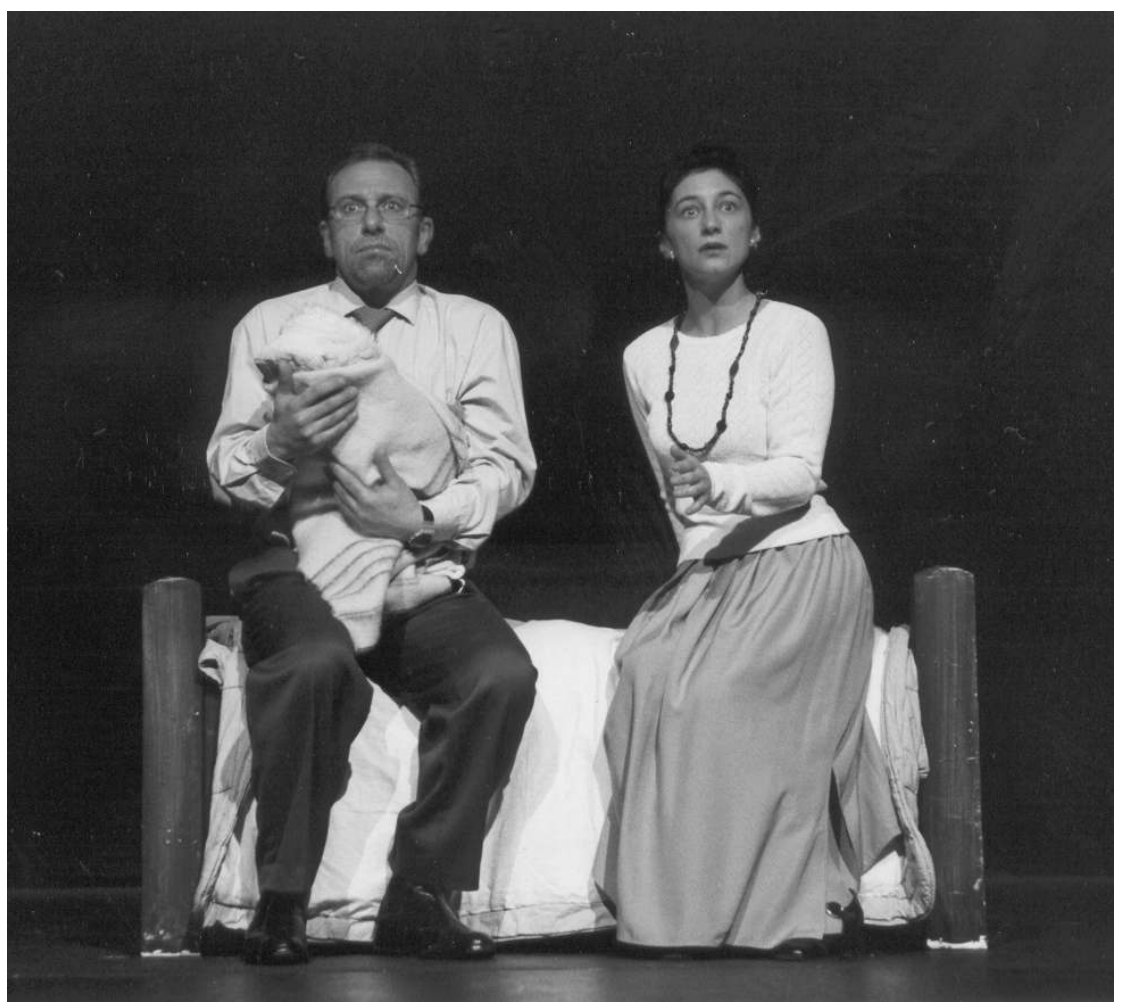

Théâtre de la Jacquerie

L. G. - J'entends bien tout Cela. mais est-ce QUe tout le monde PeUt Se Permettre DE TRAVAILler de CETte MANIÈRE ? CELA SUPPOSE du TEMPS, DONC DES MOYENS...

C. M. - Puisse se le permettre ou veuille se le permettre ? Je ne pense pas que tout le monde désire faire du laboratoire comme cela... Se donner le temps, les moyens de prendre ce temps et il faut bien le dire consentir à certains sacrifices, l'esprit «troupe » et laboratoire, la continuité et la complicité que cela suppose, et la prise de risque c'est un choix. On peut aussi choisir de monter des coups, en faisant des tours de table, des castings opportuns du " pas vu » pour être vu, etc. C'est pas trop le style de la maison... Il me semble que ce qui nous caractérise c'est plus d'abord une complicité et une culture commune, nous avons tous fait un passage chez Lecoq où Mollot a enseigné, et un sens du laboratoire ce qui ne veut pas dire du neuf à tout prix, parce que revisiter, plus exactement interroger d'anciennes formes comme la Commedia ou le Tragique ça ne nous est jamais apparu comme un travail d'archéologue. Il suffit d'ouvrir le journal de $20 \mathrm{~h}$ ou de parler au voisin du dessous, pour assister à quelques numéros de Bouffons, de Matamores, de Tragiques plus ou moins instructifs, amusants ou carrément terrifiants. À mon sens c'est un outil pour interroger la vie. Le théâtre, ce n'est ni une confidence, ni un dérivatif ! 


\section{NOTES}

1. Le théâtre de la Jacquerie a été en résidence au théâtre de Montbéliard. 